\title{
The Realization of Synchronizing Function in Roller Automatic Production Line
}

\author{
Yuan LIANG ${ }^{1}$, Jian-Ye SUN ${ }^{1, a}$ and Cheng-Feng WEI ${ }^{1}$ \\ ${ }^{1}$ Shenyang Ligong University, Engineering Technology Research Center for High Speed Cutting, \\ Shenyang 110159 China
}

Keywords: 840D System, Synchronization function, ROFFIBUS communication, Roller automatic production line.

\begin{abstract}
Roller automatic production line adopts the unit management system, and has six management control units. And each of these units takes the 840D system as the control center and adopts PROFIBUS network for communication between unit and unit. The realization of synchronization function between internal channels and the realization of synchronization function between the control units were expanded respectively.
\end{abstract}

\section{Introduction}

Rollers are main components of the transport conveyor, roller automatic production lines for parts of roller product processing and Assembly. The roller automation product line uses the unit management system, is divided into six production control unit, each control unit uses the advanced 840D system is used to control. Under control, the control unit using the 840D system of multiple groups and multiple channels technology, the control unit can be divided into several channels. Therefore, the control unit is synchronized between synchronization between and inside the channel of the roller automation production line problems needed to be resolved.

\section{Synchronization between Channels of 840D System}

In line 1, for example, 840D of 9 channels are assigned to different groups, in the automatic operation mode between each other through advanced instruction WAITM and $\mathrm{M}$ code runs synchronously.

\section{WAITM Instruction Synchronization}

Synchronous instruction WAITM (n, 2, 3) meaning: "n", waiting for the flag, "1, 2, .. "; "2,3 ", for the channel logo, representing the channel 2 and channel 3.Only channel 2 and channel 3 of the NC programs are running into the same while waiting for a sign $\mathrm{n}$, two channels at the same time continue to run down. Below to channel 2 and channel 3 of the NC programs to show the application of Directive WAITM.

Main program of channel 2:

\%_N_CHAN2_X1_MPF; \$PATH=/_N_MPF_DIR

WAITM $(1,2,3)$; Wait for synchronization flag1 of channel 3

G01 X1180 Z2663 C0; Robot runs to the upper hopper 5 imported pallets

Y-378; Manipulator downward movement

M61;

G4F1; Stop 1s

Y-10, Robot rally

WAITM $(2,2,3)$; Wait for synchronization flag2 of channel 3

G01 X-10 Z470 C90; Rotate 90 degrees to return

GOTO BB

Channel 3 the main program:

\%_N_CHAN3_X3_MPF 
; \$PATH=/_N_MPF_DIR

M60; Striker plate imports in place

BB: G0 Y400

IF \$A_IN [3] ==1 GOTO BB6; Tray material go to BB6

$\operatorname{WAITM}(1,2,3)$; Tray-free material, waiting for the synchronization flag 1 of channel 2

WAITM $(2,2,3)$; waiting for the synchronization flag2 of channel 2

BB6: STOPRE

\section{M-Code Synchronization}

In actual design and test in the found, WAITM instruction in channel number less and synchronization requirements not special closely of situation Xia using compared suitable, and in multiple channel Zhijian requirements synchronization of programming Shi, this method often is more complex, and in run in the reliability also will declined, will appeared NC program in the individual synchronization instruction was ignored of phenomenon, so at using $\mathrm{m}$ code to achieved channel of synchronization, regardless of in design and application Shang are is more convenient, and flexible and reliable. For example, to channel 40 pieces processing program in the of synchronization code M80 for cases to description its design thought, in parts processing program in the, dang program run to M80 Shi, PLC in the of corresponds to address DB24.DBX204.0 for 1, while makes DB24.DBX6.1 for 1 (NC read into ban), zhihou NC program has been in read into ban State, until other four a channel also are run to M80 Shi to reset DB24.DBX6.1, makes Channel 4 read into normal. Part of the PLC program is shown in Figure 1.

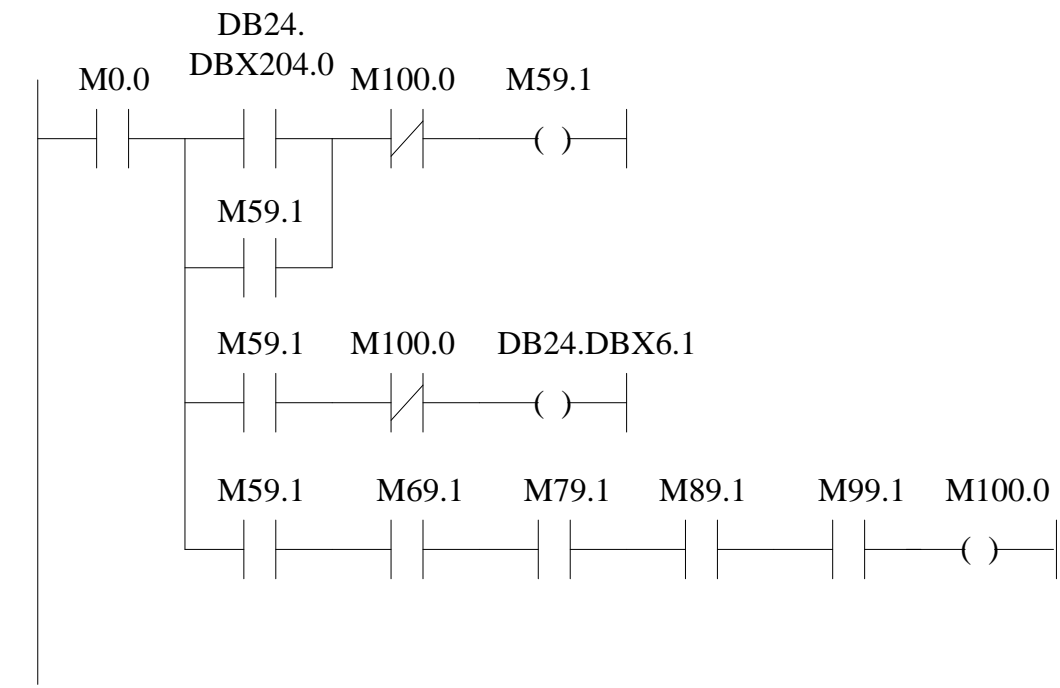

Figure 1. Implementation of synchronized code M80 in Channel 4

\section{Synchronization between Units}

Synchronization between the control unit functions mainly through network communications and software programming. Automatic production line of roller in the network structure, the control unit and set through the PROFIBUS [2] bus protocol for communication between PLC. Roller production line every production unit control system interconnected and independent of each other, in order to avoid a parking unit communications failure other unit control system, using six DP/DP in the PROFIBUS-DP network Coupler[3,4], Siemens 840D system by DP/DP each Coupler connected to the PLC, as shown in Figure 2. DP/DP Coupler coupling with electrical isolation, you can connect two different transmission speed of networks, when each child acts as a DP Slave role in the network, through continuous output from one network subnet data replication to the other input data, implement two networks Exchange data. 


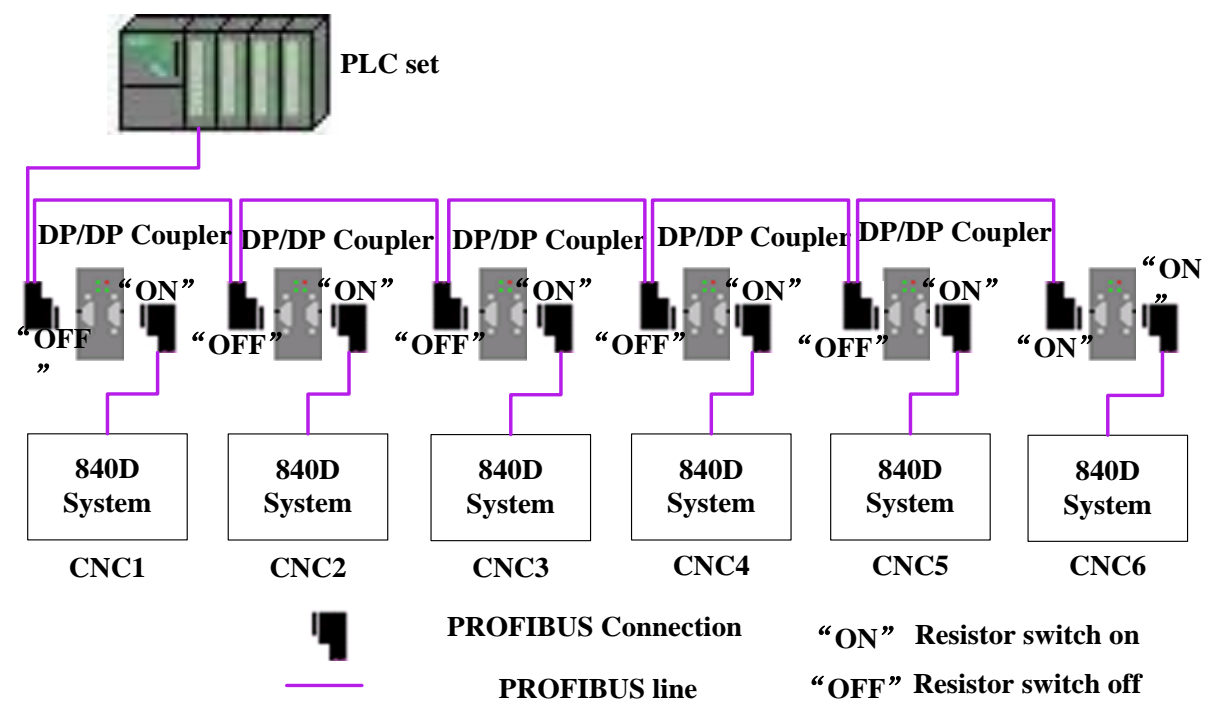

Figure 2. PROFIBUS-DP Network

\section{Network Configuration}

PLC hardware configuration is based on the hardware configuration of each unit [1], line network structure and configuration of equipment, for example in PLC hardware configuration, consideration must be given to the CPU module 315-2PN/DP must match the actual hardware, several DP/DP Coupler mailing address (Input/Output) must communicate with the corresponding unit configuration in the DP/DP Coupler address (Input/ Output) correspond to key issues. DP/DP Coupler address area address corresponds to the diagram in Figure 3.

\section{PLC set DP/DP Coupler Data \\ PLC DP/DP Coupler Data}

\begin{tabular}{|c|c|}
\hline $\begin{array}{l}\text { 6 16Bytes Input } \\
\text { IB100 } \cdots \text { IB163 } \\
1 \text { 16Bytes Output } \\
\text { QB100 } \cdots \text { QB115 }\end{array}$ & 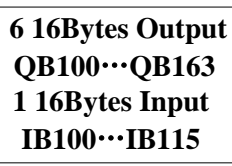 \\
\hline 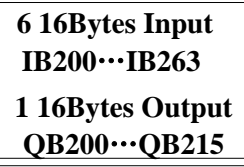 & 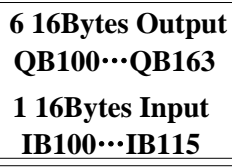 \\
\hline 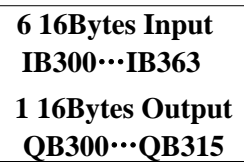 & 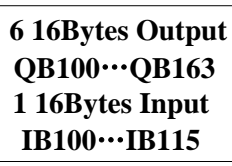 \\
\hline 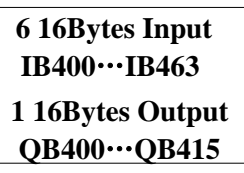 & 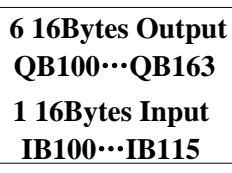 \\
\hline 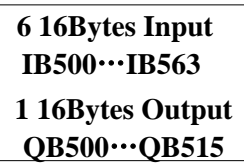 & 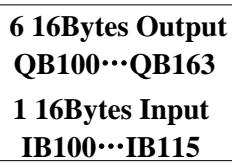 \\
\hline 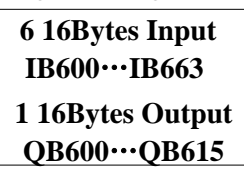 & 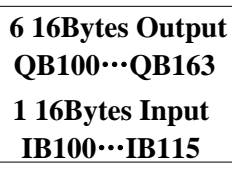 \\
\hline
\end{tabular}

Figure 3. Communication address correspondence of DP/DP Couplers 
Configuration when DP/DP Coupler, in master-slave configuration directly on individual ROFIUBS-DP bus DP/DP Coupler, a DP address is assigned to the respective DP/DP Coupler (by dial settings on the hardware) and "HW Config" hardware configuration data area good communication (Input/Output), and then save, Compiled and downloaded to the respective PLC can realize the data exchange between different PROFIBUS-DP network. DP/DP Coupler communications data in the address and signal module addresses are assigned automatically by the PLC operating system is a separate, unique address of the process image. DP/DP Coupler communications data in bytes input (Input) and output (Output) bytes and a maximum of 256 bytes, no more than 244 bytes in a single direction. As show in figure 4.

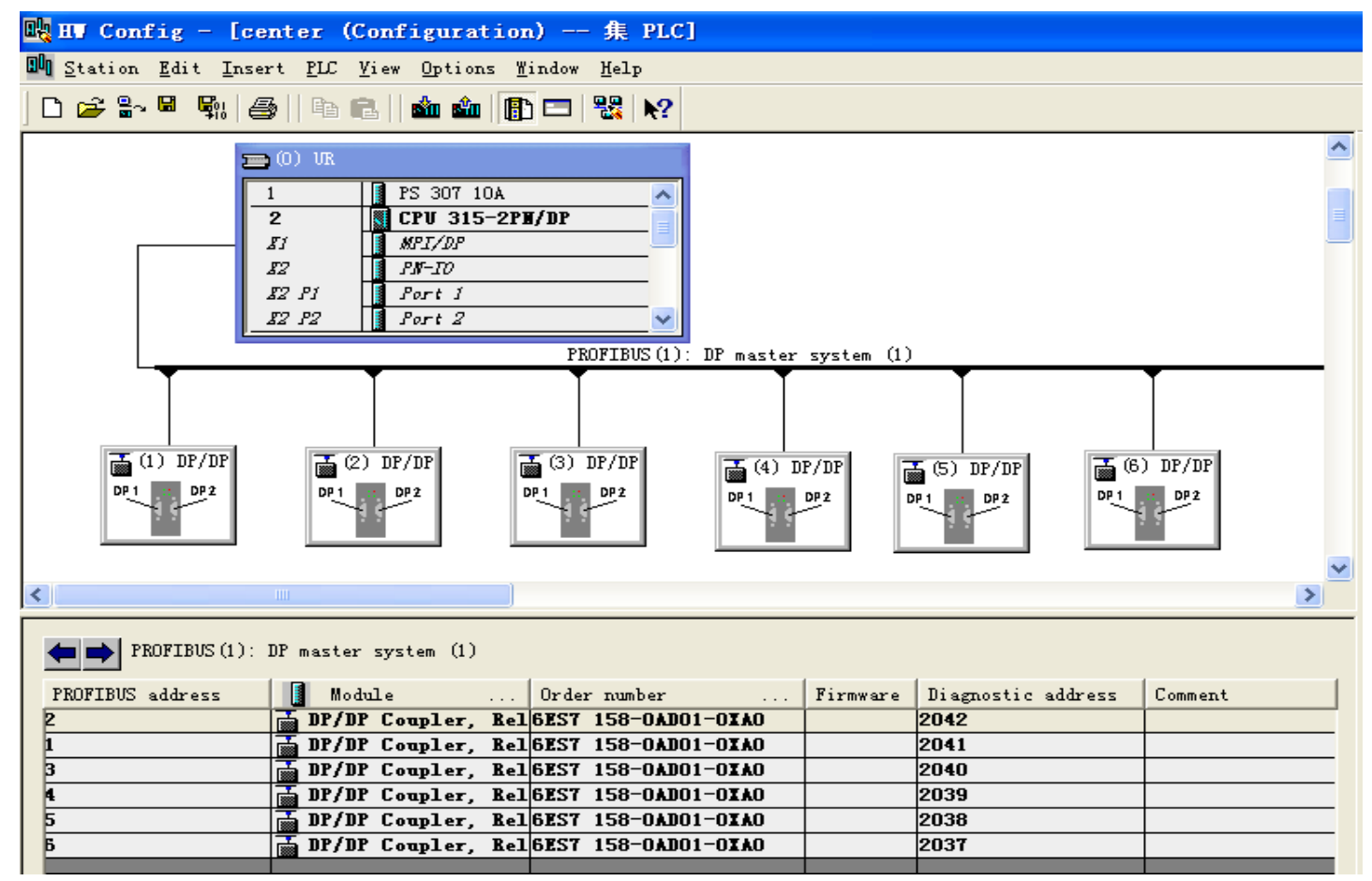

Figure 4. PLC Hardware

\section{Program Design}

In the production line, data exchange between the various production units is achieved using a data transfer instructions in PLC programs MOVE. First, the PLC DP/DP Coupler communication through each unit area "Output" can be directly read data for each unit is used for coordination between units run and then MOVE these data to be transmitted in accordance with the requirements the directive related to the specified unit DP/DP Coupler area "Input", which makes the specified unit from other unit of information and coordination of action. For example in the axis 1 production unit with shaft 2 production unit in the synchronization process, the two units will each synchronization message to PLC, Exchange respective DP/DP Coupler using the MOVE instruction information in the communications sector, then according to Exchange to synchronize information. Specifically in the PLC program for both cell synchronization information exchange procedure is shown in Figure 5, which IB163 and QB115 Exchange synchronization information for axis 1, the communication area, IB263 and QB215 for axis 2 unit Exchange sync information communication area. 


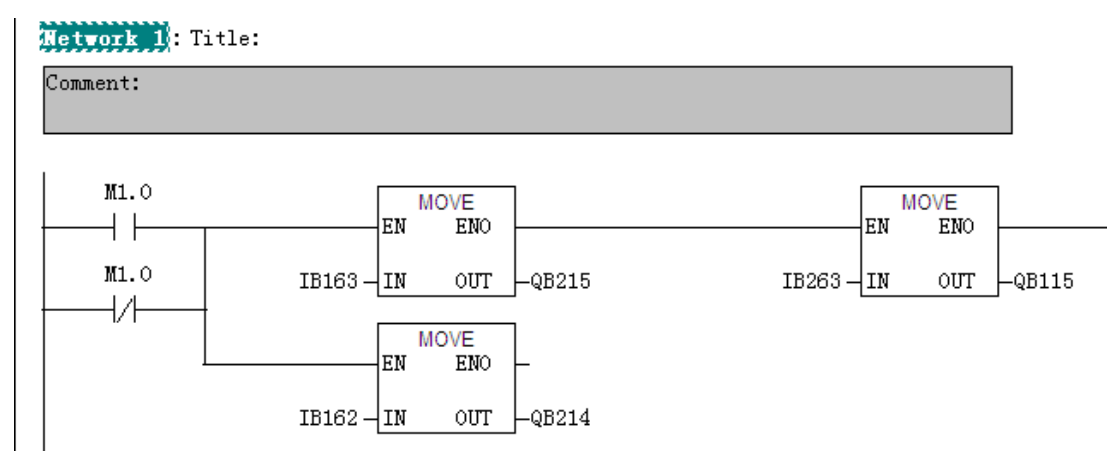

Figure 5. Realization of collaborative and synchronous data exchange between units

\section{Conclusion}

Through the use of advanced instruction in the NC program WAITM and m code, and the related function of PLC program, channel 840D system realization of synchronization function. By using the PROFIBUS network communications and software programming, achieving synchronized between cell coordination makes the automatic production line of roller become an interconnected, coordinated operation of the organic whole.

\section{References}

1. Liao Changchu. S7-300/400PLC Application technology.(2005).

2. Siemens AG . DP/DP COUPLER User Description Version V1.5. 2002.

3. C. Liao, Z.R. Zu. Siemens industrial communication network configuration programming and troubleshooting. Beijing: China machine press, 4 (9), 40-47(2009).

4. Y.P. Li, J.P. Ma. Profibus fieldbus communication technology research. Micro-computer information, (19), 59-60(2007). 